\title{
Preparation and diastereoselective methylation of enantiopure (S)-4-(1-phenylethyl)-1,4-oxazin-2-ones
}

\section{Martha Sosa-Rivadeneyra,a Leticia Quintero,*,a Cecilia Anaya de Parrodi,*,b Sylvain Bernès, ${ }^{C}$ Elena Castellanos, ${ }^{d}$ and Eusebio Juaristi,*,d}

aCentro de Investigación de la Facultad de Ciencias Químicas

Universidad Autónoma de Puebla, 72570-Puebla, México

E-mail: lquinter@siu.buap.mx

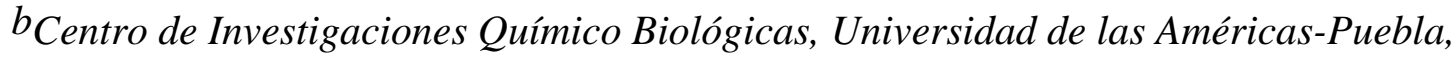

Santa Catarina Mártir, Cholula, 72820 Puebla, México

E-mail: anaya@mail.pue.udlap.mx

${ }^{C}$ Centro de Química, Instituto de Ciencias, Benemérita Universidad Autónoma de Puebla, 72000-Puebla, México,

E-mail: sylvain@eros.pquim.unam.mx

dDepartamento de Química, Centro de Investigación y de Estudios Avanzados del Instituto

Politécnico Nacional, 07000 México, D. F., México

E-mail: juaristi@chem.cinvestav.mx

(received 05 Sep 03; accepted 22 Oct 03; published on the web 23 Oct 03)

\begin{abstract}
Enantiomerically pure 1,4-oxazin-2-ones (4aS,8aS,1'S)-5, (4aR,8aR,1'S)-5, (5S,1'S)-6, (6R,1'S)7 , and $\left(5 S, 6 R, 1^{\prime} S\right)-8$ were synthesized from the corresponding $\beta$-aminoalcohols in the presence of glyoxal, in good yields (65-86\%). These 1,4-oxazin-2-ones were methylated to afford the corresponding alkylated derivatives (3R,4a $S, 8 \mathrm{a} S, 1$ 'S)-9, (3S,4aR,8aR,1'S)-9, (3R,5S,1'S)-10, $(3 S, 5 S, 6 R, 1 ' S)-12$ in moderate yields (25-60\%), and low to excellent diastereoselectivities (up to ca. $100 \%$ ). A reasonable interpretation of the experimental results was reached by molecular modeling studies at the AM1 semiempirical level.
\end{abstract}

Keywords: $\quad 1,4-O x a z i n-2-o n e s, \quad \beta$-aminoalcohols, $\quad$ diastereoselective alkylation, $\alpha$ phenylethylamine

\section{Introduction}

In recent years, the preparation of enantiopure six-membered heterocycles containing oxygen and nitrogen has received significant attention, owing to their high potential as chiral substrates. ${ }^{1}$ 
In particular, 1,4-oxazin-2-ones have been used in the asymmetric synthesis of $\alpha$-amino acids, $^{2}$ and other natural products. ${ }^{3}$

Among the various methods developed for the preparation of 1,4-oxazin-2-ones, ${ }^{4}$ a widely used protocol involves the condensation reaction of $\beta$-aminoalcohols with glyoxal. ${ }^{5}$

Motivated by the demonstrated efficiency of $(R)$ - and $(S)$ - $\alpha$-phenylethylamine in the preparation of enantiopure compounds, ${ }^{6}$ we decided to undertake the synthesis of novel (S)-4-(1phenylethyl)-1,4-oxazin-2-ones $\quad\left(4 \mathrm{a} S, 8 \mathrm{a} S, 1^{\prime} S\right)-5, \quad\left(4 \mathrm{a} R, 8 \mathrm{a} R, 1^{\prime} S\right)-5, \quad\left(5 S, 1^{\prime} S\right)-6, \quad(6 R, 1$ ' $S)-7$, (5S,6R,1'S)-8 (Scheme 1), from the corresponding chiral $N$-[(S)- $\alpha$-phenyl-ethyl]- $\beta$-aminoalcohols $\left(1 S, 2 S, 1^{\prime} S\right)-\mathbf{1},\left(1 R, 2 R, 1^{\prime} S\right)-\mathbf{1},\left(2 S, 1^{\prime} S\right)-\mathbf{2},(1 R, 1 ' S)-\mathbf{3}$, and $\left(1 R, 2 S, 1^{\prime} S\right)-\mathbf{4}^{7}$

\section{Results and Discussion}

The enantiopure 1,4-oxazin-2-ones of interest were prepared by the condensation of glyoxal and the corresponding $N$-[(S)- $\alpha$-phenyl-ethyl]- $\beta$-aminoalcohols. ${ }^{7-10}$ Following chromatographic purification, the morpholinones were fully characterized.<smiles>C[C@H](N[C@H]1CCCC[C@@]1(O)c1ccccc1)c1ccccc1</smiles>

$(1 S, 2 S, 1 ' S)-1$
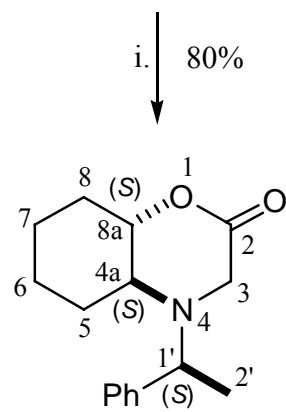

$\left(4 \mathrm{aS}, 8 \mathrm{aS}, 1{ }^{\prime} S\right)-5$

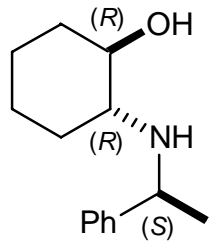

$(1 R, 2 R, 1 ' S)-1$
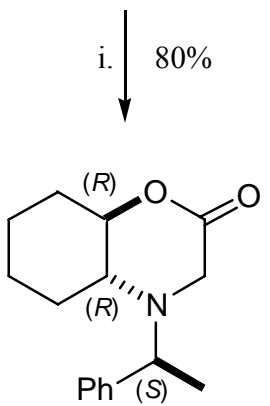

$(4 \mathrm{a} R, 8 \mathrm{a} R, 1 \mathrm{~S})-5$

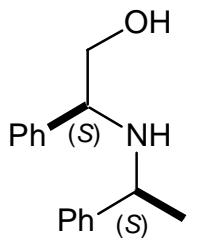

$(2 S, 1 ' S)-2$

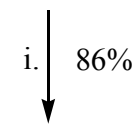

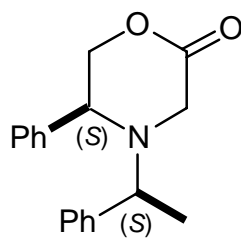

$(5 S, 1 ' S)-6$

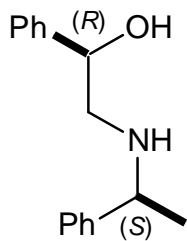

$(1 R, 1 ' S)-3$
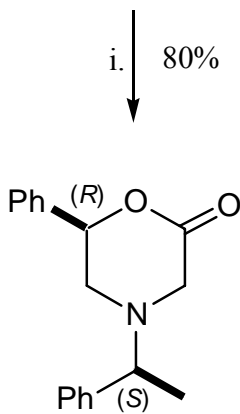

$(6 R, 1 ' S)-7$

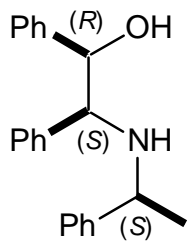

$(1 R, 2 S, 1 ' S)-4$

Scheme 1. Reagents and conditions: i. Glyoxal/THF, 2h, reflux.

The high acidity of the hydrogen atoms at $\mathrm{C}(3)$ in the morpholinone ring allows for alkylation. Indeed, in the presence of lithium diisopropylamide (LDA) and methyl iodide, the 1,4-oxazin-2-ones 5-8 afforded the corresponding methylated compounds.

Highest diastereoselectivities (d.e. $=88-100 \%$ ) were obtained with the more substituted 1,4oxazin-2-ones (4aS,8aS,1'S)-5, (4aR,8aR,1'S)-5, and (5S,6R,1'S)-8 (entries 1, 2 and 5, Table 1). 
On the other hand, the monosubstituted 1,4-oxazin-2-ones, (5S,1'S)-6 and (6R,1'S)-7, afforded the methylated products with low diastereoselectivities (d.e. $=14-29 \%$ ) (entries 3 and 4, Table $1)$.

Table 1. Diastereoselective methylation of (S)-4-(1-phenylethyl)-1,4-oxazin-2-ones 5-8

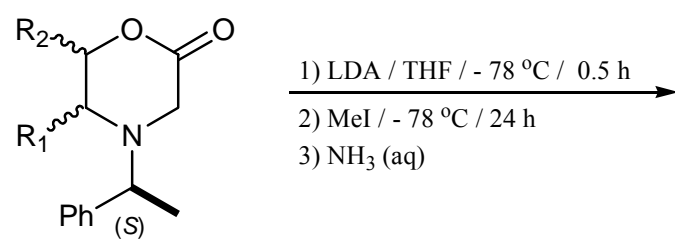

5-8<smiles>[R20]C1OC(=O)[C@@H](C)N([C@H](C)c2ccccc2)[C@H]1[R14]</smiles>

9-11

\begin{tabular}{|c|c|c|c|c|c|c|}
\hline Entry & 1,4-Oxazin-2-one & $\mathrm{R}_{1}$ & $\mathrm{R}_{2}$ & $\begin{array}{l}\text { Yield } \\
(\%)^{\mathrm{a}}\end{array}$ & $\begin{array}{c}\text { Diastereomer } \\
\text { ratio }^{b}\end{array}$ & Major product \\
\hline 1 & $\left(4 \mathrm{a} S, 8 \mathrm{a} S, 1^{\prime} S\right)-\mathbf{5}$ & \multicolumn{2}{|c|}{$-\mathrm{C}_{4} \mathrm{H}_{8-}$} & 80 & $94: 6$ & $\left(3 R, 4 \mathrm{a} S, 8 \mathrm{a} S, 1^{\prime} S\right)-\mathbf{9}$ \\
\hline 2 & $(4 \mathrm{a} R, 8 \mathrm{a} R, 1 ' S)-5$ & \multicolumn{2}{|c|}{$-\mathrm{C}_{4} \mathrm{H}_{8}-$} & 60 & $98: 2$ & $(3 S, 4 \mathrm{a} R, 8 \mathrm{a} R, 1 ' S)-\mathbf{9}$ \\
\hline 3 & $\left(5 S, 1^{\prime} S\right)-6$ & $\mathrm{Ph}-$ & $\mathrm{H}-$ & 34 & $61: 39$ & $\left(3 R, 5 S, 1^{\prime} S\right)-\mathbf{1 0}$ \\
\hline 4 & $\left(6 R, 1^{\prime} S\right)-7$ & $\mathrm{H}-$ & $\mathrm{Ph}-$ & 60 & $57: 43$ & $\left(3 R S, 6 R, 1^{\prime} S\right)-11^{\mathrm{c}}$ \\
\hline 5 & $(5 S, 6 R, 1 ' S)-8$ & $\mathrm{Ph}-$ & $\mathrm{Ph}-$ & 59 & $\sim 100: 0$ & $(3 S, 5 S, 6 R, 1 ' S)-\mathbf{1 2}$ \\
\hline
\end{tabular}

${ }^{a}$ After chromatographic separation on silica gel (eluent: petroleum ether/ethyl acetate, 7:1). ${ }^{b}$ Determined by HPLC with a C18 column; eluent: methanol/water, 70:30; flow: $1 \mathrm{~mL} / \mathrm{min}$. ${ }^{\mathrm{c}}$ The diastereoisomeric mixture was not separated.

The assignment of the absolute configuration of the new stereogenic center in the major methylated products (with the exception of 11) was possible by X-ray diffraction analysis of crystalline (3R,4aS,8aS,1'S)-9, (3S,4aR,8aR,1'S)-9, (3R,5S,1'S)-10, and $\left(3 S, 5 S, 6 R, 1^{\prime} S\right)-\mathbf{1 2},{ }^{11}$ which were isolated by flash chromatography, and successively recrystallized (petroleum ether/dichloromethane). Scheme 2 shows the molecular structure and solid state conformation of these compounds. 
(a)<smiles>C[C@H]1C(=O)O[C@]2(Cl)CCCC[C@@H]2N1[C@H](C)c1ccccc1</smiles>

$(3 R, 4 \mathrm{a} S, 8 \mathrm{a} S, 1 ' S)-9$

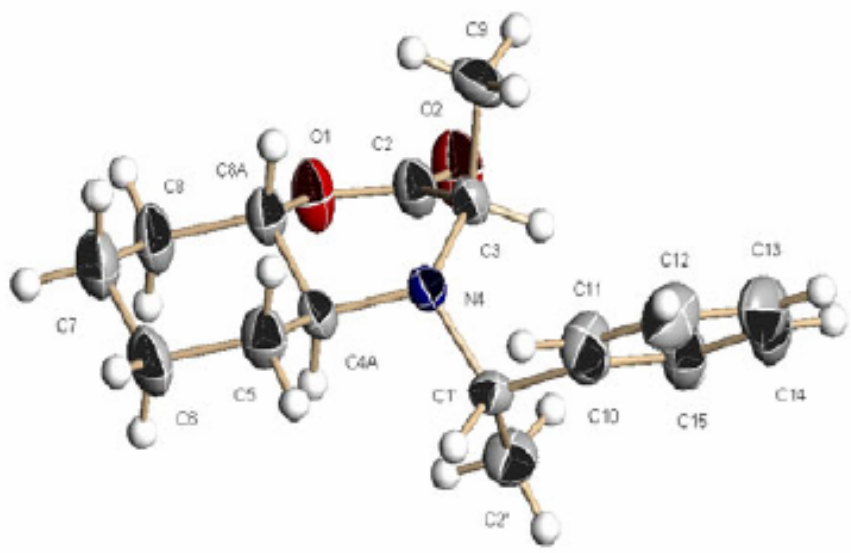

(b)<smiles>C[C@@H](c1ccccc1)N1[C@H]2CCCC[C@H]2OC(=O)[C@H]1C</smiles>

$(3 S, 4 \mathrm{a} R, 8 \mathrm{a} R, 1 ' S)-9$

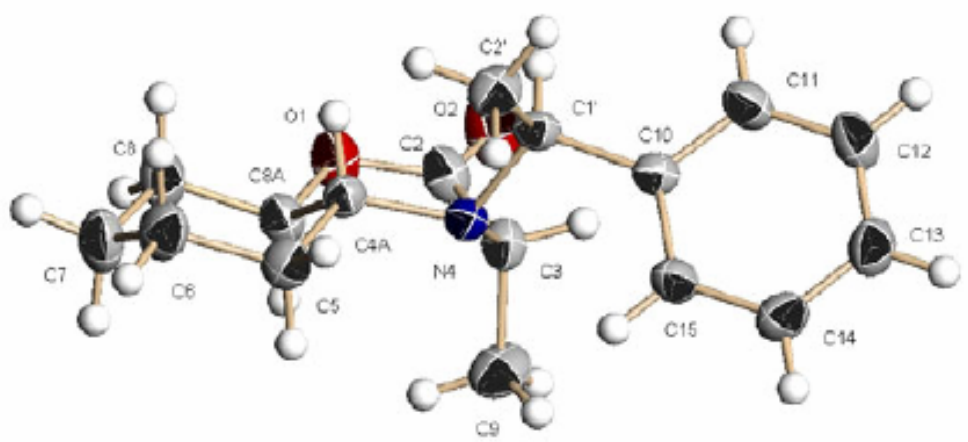

(c)<smiles>C[C@H]1C(=O)OC[C@@H](c2ccccc2)N1[C@H](C)c1ccccc1</smiles>

$(3 R, 5 S, 1 ' S)-10$

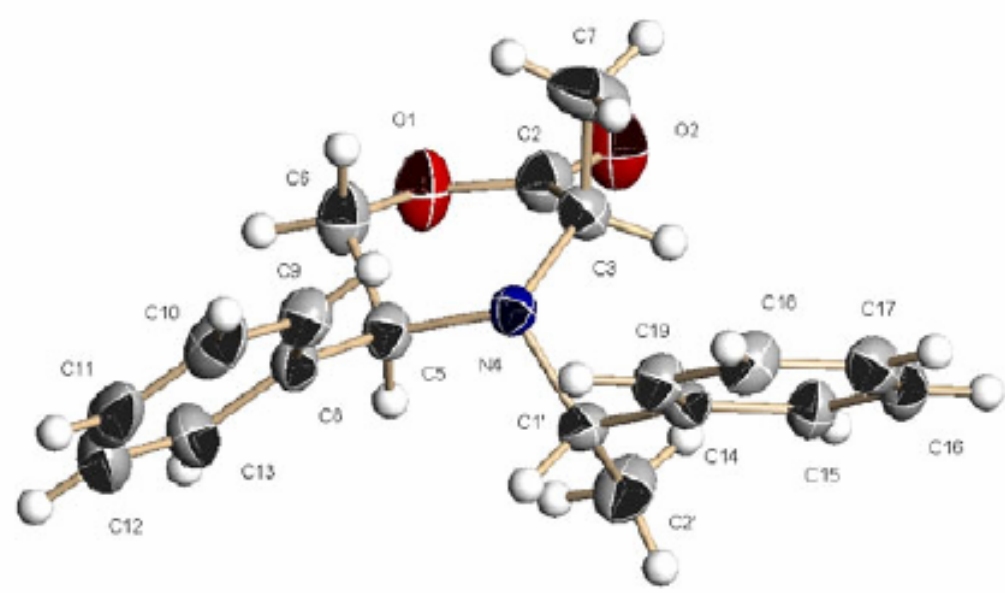


(d)

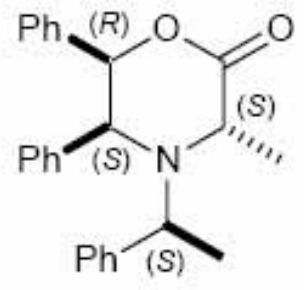

$(3 S, 5 S, 6 R, 1 ' S)-12$

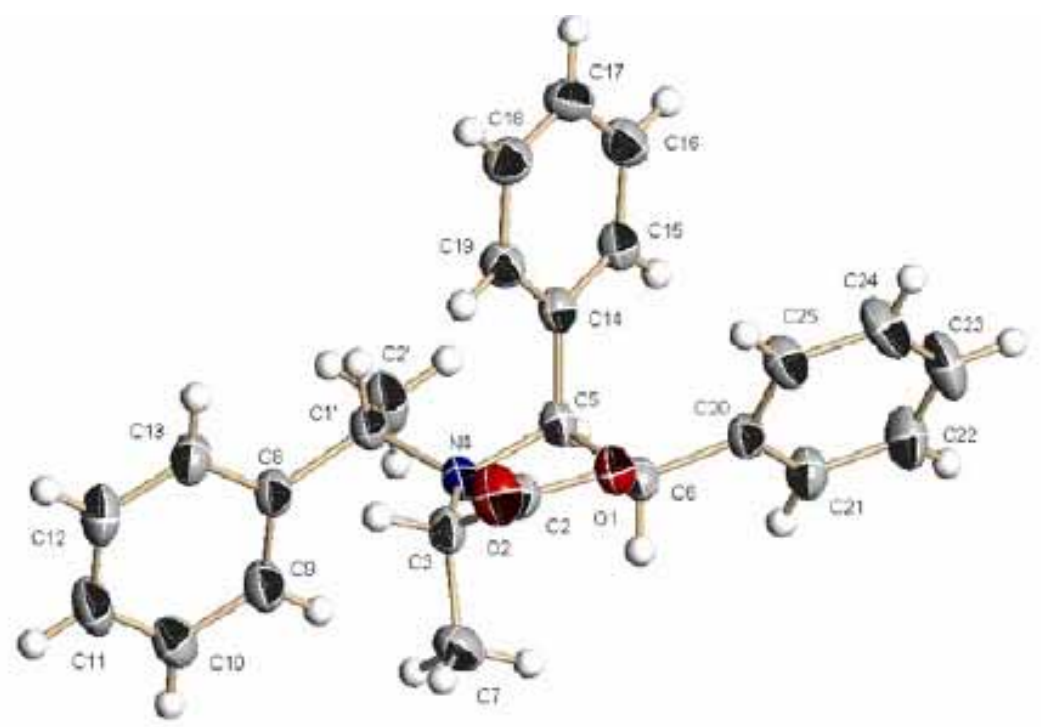

Scheme 2. Structure and solid-state conformation of (a) $\left(3 R, 4 \mathrm{a} S, 8 \mathrm{a} S, 1^{\prime} S\right)-\mathbf{9}$, $\left(3 S, 4 \mathrm{a} R, 8 \mathrm{a} R, 1^{\prime} S\right)-\mathbf{9}$, (c) $\left(3 R, 5 S, 1^{\prime} S\right)-\mathbf{1 0}$, and (d) $\left(3 S, 5 S, 6 R, 1^{\prime} S\right)-12$.

The high diastereoselectivity observed in the methylation reactions of enolates ( $4 \mathrm{a} S, 8 \mathrm{a} S, 1$ ' $S$ )5-Li, $\left(4 \mathrm{a} R, 8 \mathrm{a} R, 1^{\prime} S\right)-5-\mathrm{Li}$, and $\left(5 S, 6 R, 1^{\prime} S\right)-8-\mathrm{Li}$ (entries 1, 2, and 5 in Table 1) can be understood in the light of semiempirical AM1 method, accessible in the program Hyperchem 5.1 (Hypercube, Inc.). ${ }^{12}$

Indeed, the structures of lowest energy for these enolates (Scheme 3) clearly show that the $\mathrm{N}$ phenylethyl substituent in $\left(4 \mathrm{aS}, 8 \mathrm{a} S, 1^{\prime} S\right)$-5-Li sterically hinders the enolate's $\mathrm{Si}$ face, which is in agreement with the predominant formation of the (3R) product, in $94 \% \mathrm{ds} .^{13}$

In contrast, the $N$-phenylethyl group in enolate $\left(4 \mathrm{a} R, 8 \mathrm{a} R, 1^{\prime} S\right)-5$-Li adopts a new orientation, dictated by the $(R, R)$-configured ring carbons $4 \mathrm{a}$ and $8 \mathrm{a}$, that now hinders alkylation on the $R e$ face. (Scheme 3). This observation is again in line with the experimentally checked large predominance of diastereomeric product $\left(3 S, 4 \mathrm{a} R, 8 \mathrm{a} R, 1^{\prime} S\right)-\mathbf{9}$.

Scheme 3. Lowest-energy structures calculated (AM1

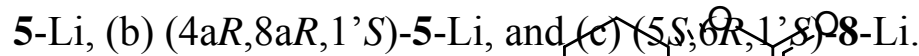
(a) $(4 \mathrm{a}, 8 \mathrm{aS}, 1 \mathrm{~S})-5-\mathrm{Li}$

By the same token, the calculated Nlowest-eners presents a pseudo-axial orientation of $\mathrm{C}(5)-\mathrm{ph}$ electrophile to the Re face. (Scheme 3 ). This effect annears to ne most significant since experimentally the reaction does proceed with near p methyl iodide addition on the enolate's $S i$ face, $(3 S, 5 S, 6$ i

(b) $\left(4 \mathrm{a} R, 8 \mathrm{a} R, 1^{\prime} S\right)-5-\mathrm{Li}$<smiles>O=C1CN[C@@H]2CCCC[C@H]2O1</smiles><smiles>C[C](C)c1ccccc1</smiles>

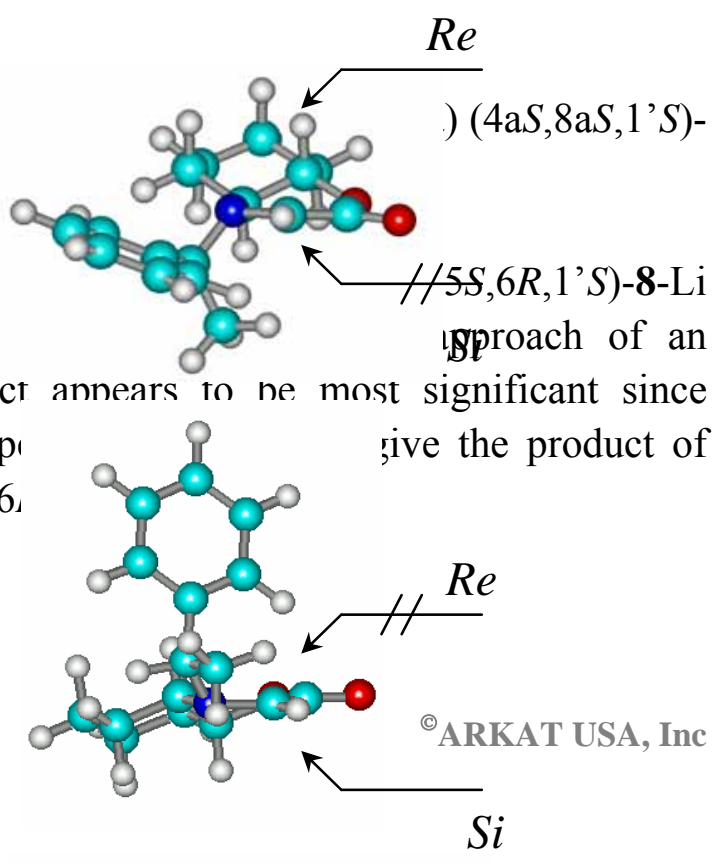


The low diastereoselectivity observed in the methylation reaction of enolate $\left(5 S, 1^{\prime} S\right)-6-\mathrm{Li}$ can be explained upon consideration of the fact that molecular modeling (AM1 semiempirical method) suggests three conformations of comparable energy (Scheme 4). Most relevant, one of these conformers (I in Scheme 4) favors alkylation on the Si face of enolate 6-Li, whereas the second lowest-energy conformer (II in Scheme 4) favors addition on the opposite Re face. Finally, both enolate faces in the third low-energy conformer (III in Scheme 4) seem to be equally hindered. Thus, competition between the reactive enolate conformers I and II is apparently responsible for the poor diastereoselectivity in the methylation reaction, as registered in entry 3 of Table 1.

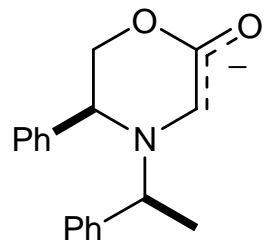

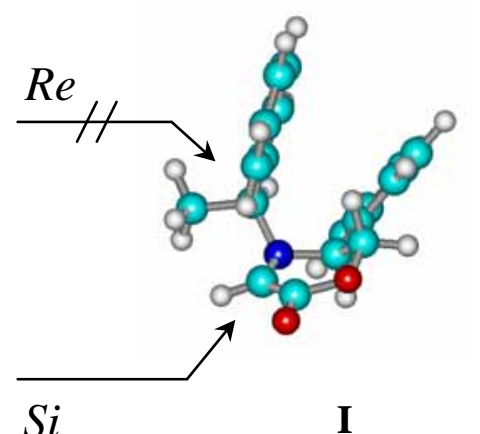

$\mathrm{E}_{\mathrm{I}}=0.00 \mathrm{kcal} / \mathrm{mol}$ $\left(5 S, 1^{\prime} S\right)-6-\mathrm{Li}$

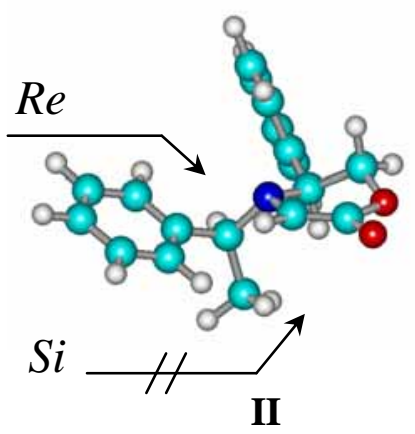

$\mathrm{E}_{\mathrm{II}}=+0.38 \mathrm{kcal} / \mathrm{mol}$

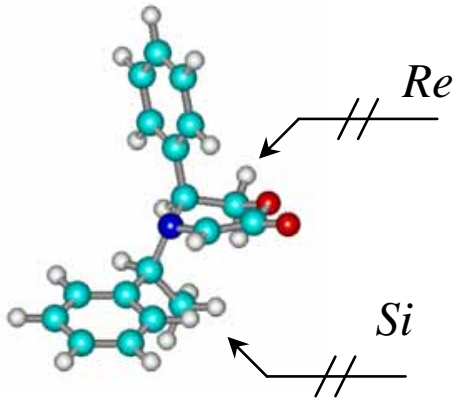

III

$\mathrm{E}_{\mathrm{III}}=+0.72 \mathrm{kcal} / \mathrm{mol}$

Scheme 4. Lowest-energy conformers of (5S,1'S)-6-Li and their relative energy, as calculated by the semiempirical AM1 method. ${ }^{14}$

A relative flat potential energy profile is also calculated (AM1 semiempirical method) for enolate (6R,1'S)-7-Li. Indeed, four stable conformers of comparable energy are detected. As can be appreciated in Scheme 5, either the Re or Si face becomes favorable for alkylation depending on the enolate conformation, affording little diastereoselectivity (entry 4 in Table 1). 
<smiles>CC1=CN([C@H](C)c2ccccc2)[C@@H](c2ccccc2)OC1=O</smiles>

(a)

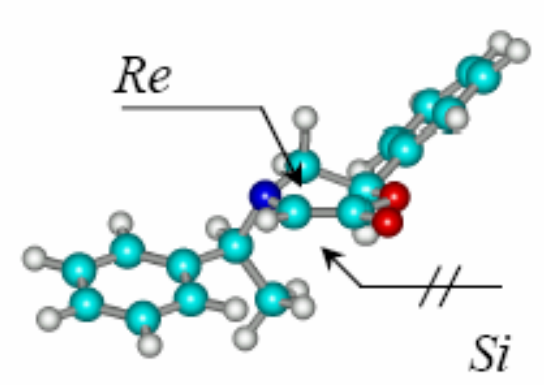

$$
\mathrm{E}=0.00 \mathrm{kcal} / \mathrm{mol}
$$

(b)

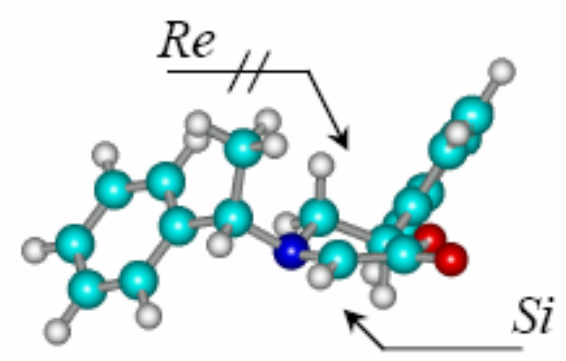

$\mathrm{E}=+1.28 \mathrm{kcal} / \mathrm{mol}$ (c)

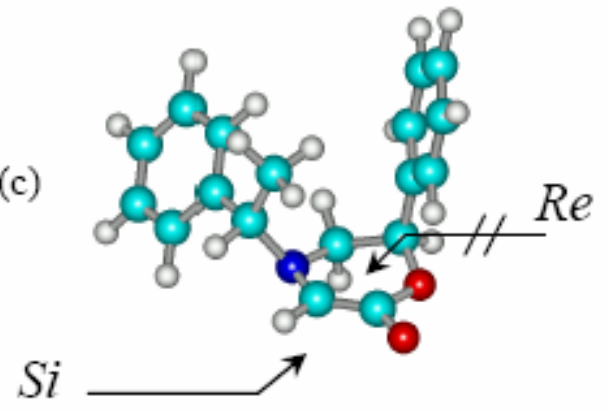

$\mathrm{E}=+1.24 \mathrm{kcal} / \mathrm{mol}$

(d)

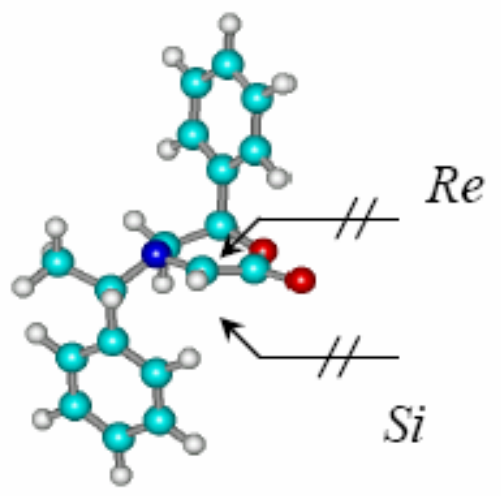

$\mathrm{E}=+1.37 \mathrm{kcal} / \mathrm{mol}$

Scheme 5. Lowest-energy conformers of $\left(6 R, 1^{\prime} S\right)-7-\mathrm{Li}$ and their relative energy, according to semiempirical AM1 calculations. ${ }^{14}$

\section{Conclusions}

In summary, novel chiral 1,4-oxazin-2-ones (4aS,8aS,1'S)-5, (4aR,8aR,1'S)-5, (5S,1'S)-6, $\left(6 R, 1^{\prime} S\right)-7$, and $\left(5 S, 6 R, 1^{\prime} S\right)-8$ were prepared in good yield from the condensation reaction between the corresponding $\beta$-aminoalcohol and glyoxal. Alkylation of these oxazinones was possible via enolate formation with LDA, and proceeds with high diastereoselectivity with enolates $\quad\left(4 \mathrm{a} S, 8 \mathrm{a} S, 1^{\prime} S\right)-5-\mathrm{Li}, \quad\left(4 \mathrm{a} R, 8 \mathrm{a} R, 1^{\prime} S\right)-5-\mathrm{Li}, \quad$ and $\quad\left(5 S, 6 R, 1{ }^{\prime} S\right)-8-\mathrm{Li} . \quad$ A reasonable 
interpretation of the experimental results was reached by molecular modeling studies at the AM1 semiempirical level.

\section{Experimental Section}

General Procedures. Melting points were determined with a Fischer Jones apparatus, and are uncorrected. Column chromatography was performed on silica gel (Merck 60, 70-230 mesh). Reactions were monitored by TLC on aluminium backed plates of silica gel 60 F254 (Merck, 0.2 $\mathrm{mm})$. NMR spectra were recorded on a Varian $400\left(9.4\right.$ Tesla, $400.13 \mathrm{MHz}$ for ${ }^{1} \mathrm{H}$, and 100.62 $\mathrm{MHz}$ for $\left.{ }^{13} \mathrm{C}\right)$ spectrometer. Chemical shifts $(\delta$ in $\mathrm{ppm})$ are given from internal $\mathrm{CDCl}_{3}(7.26$ ppm) for ${ }^{1} \mathrm{H} \mathrm{NMR}, \mathrm{CDCl}_{3}(77.0 \mathrm{ppm})$ for ${ }^{13} \mathrm{C} \mathrm{NMR}$. Coupling constants ( $J$ in $\mathrm{Hz}$ ) are accurate to $\pm 0.2 \mathrm{~Hz}$ for ${ }^{1} \mathrm{H}$ and $\pm 0.6 \mathrm{~Hz}$ for ${ }^{13} \mathrm{C}$. HPLC experiments were carried out on a Controller Waters 600 equipped with photodiode array detector Waters 996.

\section{General procedure for the preparation of 1,4-oxazin-2-ones}

A one-necked flask fitted with a magnetic stirrer was charged with the corresponding $\beta$ aminoalcohol $(4.55 \mathrm{mmol})$ in THF $(20 \mathrm{~mL})$. Then, an aqueous solution of glyoxal $(40 \%, 0.66$ $\mathrm{mL}, 4.55 \mathrm{mmol}$ ) was added and the reaction mixture was heated to reflux for $2 \mathrm{~h}$. The solution was extracted with $\mathrm{CH}_{2} \mathrm{Cl}_{2}(3 \times 10 \mathrm{~mL})$ and water $(50 \mathrm{~mL})$. The combined organic layers were dried with anhydrous $\mathrm{Na}_{2} \mathrm{SO}_{4}$, filtered, and evaporated. The crude product was purified by flash chromatography on silica gel (eluent: petroleum ether/ethyl acetate, 7:1).

(4aS,8aS,1'S)-4-(1-Phenylethyl)-octahydro-benzo[1,4]oxazin-2-one (5). White solid (80\% yield), mp 79-80 ${ }^{\circ} \mathrm{C} ;[\alpha]_{\mathrm{D}}{ }^{28}=+21.2(\mathrm{c}=1, \mathrm{EtOH}) ;{ }^{1} \mathrm{H} \mathrm{NMR}: \delta 1.31(\mathrm{~d}, J=6.9 \mathrm{~Hz}, 3 \mathrm{H}), 1.48(\mathrm{~m}$, $2 \mathrm{H}), 1.52(\mathrm{~m}, 2 \mathrm{H}), 1.84(\mathrm{~m}, 2 \mathrm{H}), 2.18(\mathrm{~m}, 2 \mathrm{H}), 2.52(\mathrm{~m}, 1 \mathrm{H}), 3.20(\mathrm{~d}, J=1.8 \mathrm{~Hz}, 2 \mathrm{H}), 4.08(\mathrm{~m}$, 1H), 4.28 (q, $J=6.9 \mathrm{~Hz}, 1 \mathrm{H}), 7.30(\mathrm{~m}, 5 \mathrm{H}) ;{ }^{13} \mathrm{C}$ NMR: $\delta 8.8,23.8,24.3,28.7,30.8,46.9,54.1$, 59.7, 82.3, 127.1, 127.8, 128.3, 141.8, 169.8. Anal. Calcd for $\mathrm{C}_{16} \mathrm{H}_{21} \mathrm{NO}_{2}$ (259.34): C, 74.08; $\mathrm{H}$, 8.16. Found: C, 74.30; H, 8.24.

(4aR,8aR,1'S)-4-(1-Phenylethyl)octahydrobenzo[1,4]oxazin-2-one (5). White solid (80\% yield), mp $96-98{ }^{\circ} \mathrm{C} ;[\alpha]_{\mathrm{D}}{ }^{28}=+48.9(\mathrm{c}=1, \mathrm{EtOH}) ;{ }^{1} \mathrm{H} \mathrm{NMR}: \delta 1.27(\mathrm{~m}, 4 \mathrm{H}), 1.44(\mathrm{~d}, J=7.0 \mathrm{~Hz}$, $3 \mathrm{H}), 1.78(\mathrm{~m}, 2 \mathrm{H}), 2.07(\mathrm{~m}, 2 \mathrm{H}), 2.30(\mathrm{~m}, 1 \mathrm{H}), 3.17(\mathrm{~d}, J=17.5 \mathrm{~Hz}, 1 \mathrm{H}), 3.69$ (d, J = $17.5 \mathrm{~Hz}$, 1H), 4.03 (m, 1H), 4.18 (q, 1H), 7.2-7.38 (m, 5H); ${ }^{13} \mathrm{C}$ NMR: $\delta$ 18.4, 23.9, 24.3, 29.9, 31.2, 48.6, $56.7,59.8,81.9,127.3,127.7,128.2,139.1,169.5$. HRMS (FAB) m/z found 282.1464 [M+Na] $]^{+}$, calcd for $\mathrm{C}_{16} \mathrm{H}_{21} \mathrm{NO}_{2} \mathrm{Na} 282.1470$.

(5S,1'S)-5-Phenyl-4-(1-phenylethyl)-1,4-oxazin-2-one (6). White solid (86\% yield), mp 87-88 ${ }^{\circ} \mathrm{C}$; $[\alpha]_{\mathrm{D}}{ }^{28}=-37.1(\mathrm{c}=1, \mathrm{EtOH}) ;{ }^{1} \mathrm{H}$ NMR: $\delta 1.25(\mathrm{~d}, J=7.0 \mathrm{~Hz}, 3 \mathrm{H}), 3.36(\mathrm{~s}, 2 \mathrm{H}), 3.94(\mathrm{q}, 1 \mathrm{H})$, $4.04(\mathrm{~m}, J=5.1 \mathrm{~Hz}, 1 \mathrm{H}), 4.32(\mathrm{~m}, 2 \mathrm{H}), 7.20-7.38(\mathrm{~m}, 10 \mathrm{H}) ;{ }^{13} \mathrm{C} \mathrm{NMR}: \delta 9.4,46.8,55.8,60.2$, $73.0,127.4,127.7,128.0,128.4,128.8,129.9,136.9,141.4,156.5,169.5$. HRMS (FAB) m/z found $282.1485[\mathrm{M}+\mathrm{H}]^{+}$, calcd for $\mathrm{C}_{18} \mathrm{H}_{20} \mathrm{NO}_{2} 282.1494$.

(6R,1'S)-6-Phenyl-4-(1-phenylethyl)-1,4-oxazin-2-one (7). White solid (76\% yield), mp 90-92 ${ }^{\circ} \mathrm{C}$; $[\alpha]_{\mathrm{D}}{ }^{28}=-29.0\left(\mathrm{c}=1.02, \mathrm{CHCl}_{3}\right) ;{ }^{1} \mathrm{H} \mathrm{NMR}: \delta 1.37(\mathrm{~d}, J=7.0 \mathrm{~Hz}, 3 \mathrm{H}), 2.45(\mathrm{dd}, J=3.3 \mathrm{~Hz}, J=$ 
$1.7 \mathrm{~Hz}, 1 \mathrm{H}), 3.13(\mathrm{~d}, J=17.5 \mathrm{~Hz}, 1 \mathrm{H}), 3.16(\mathrm{dd}, J=3.3 \mathrm{~Hz}, J=1.7 \mathrm{~Hz}, 1 \mathrm{H}) 3.46(\mathrm{q}, J=7.0 \mathrm{~Hz}$, $1 \mathrm{H}), 3.56(\mathrm{dd}, J=1.7 \mathrm{~Hz}, 1 \mathrm{H}), 5.50(\mathrm{dd}, J=3.3 \mathrm{~Hz}, J=1.7 \mathrm{~Hz}, 1 \mathrm{H}), 7.19-7.37(\mathrm{~m}, 10 \mathrm{H}) ;{ }^{13} \mathrm{C}$ NMR: $\delta 19.5,53.4,53.9,64.0,81.2,126.3,127.3,126.4,127.7,126.9,128.6,128.7,128.8$, 128.9, 128.9, 138.0, 141.7, 168.5; HRMS (FAB) $\mathrm{m} / \mathrm{z}$ found $304.1309[\mathrm{M}+\mathrm{Na}]^{+}$, calcd for $\mathrm{C}_{18} \mathrm{H}_{19} \mathrm{NO}_{2} \mathrm{Na} 304.1314$.

(5S,6R,1'S)-5,6-Diphenyl-4-(1-phenylethyl)-1,4-oxazin-2-one (8). White solid (65\% yield), mp $157-158{ }^{\circ} \mathrm{C} ;[\alpha]_{\mathrm{D}}{ }^{28}=-49.7\left(\mathrm{c}=1.12, \mathrm{CHCl}_{3}\right) ;{ }^{1} \mathrm{H}$ NMR: $\delta 1.39(\mathrm{~d}, J=6.6 \mathrm{~Hz}, 3 \mathrm{H}), 3.51(\mathrm{~d}, J=$ $17.64 \mathrm{~Hz}, 1 \mathrm{H}), 3.68$ (d, $J=17.24 \mathrm{~Hz}, 1 \mathrm{H}), 3.78(\mathrm{q}, 1 \mathrm{H}), 4.41(\mathrm{~d}, J=3.8 \mathrm{~Hz}, 1 \mathrm{H}), 5.70(\mathrm{~d}, 1 \mathrm{H})$, 6.89-7.32( m, 15H); ${ }^{13} \mathrm{C}$ NMR: $\delta$ 15.9, 48.6, 59.3, 63.5, 82.7, 126.8, 127.6, 127.9, 127.9, 128.0, 128.2, 128.6, 129.5, 135.6, 141.9, 170.1; HRMS (FAB) m/z found $380.1634[\mathrm{M}+\mathrm{Na}]^{+}$, calcd for $\mathrm{C}_{24} \mathrm{H}_{23} \mathrm{NO}_{2} \mathrm{Na} 380.1627$.

\section{General procedure for the C(3)-methylation of 1,4-oxazin-2-ones 5-8}

A dry two-necked flask fitted with a magnetic stirrer and a low temperature thermometer was charged with the corresponding 1,4-oxazin-2-one $(1.15 \mathrm{mmol})$ in dry THF $(10 \mathrm{~mL})$ under argon. The solution was cooled at $-78{ }^{\circ} \mathrm{C}$ and lithium diisopropylamide in heptane/THF/ethylbenzene solution $(2 \mathrm{M}, 1.72 \mathrm{~mL}, 3.47 \mathrm{mmol})$ was added. The reaction mixture was stirred for 30 minutes at $-78{ }^{\circ} \mathrm{C}$ and methyl iodide $(0.23 \mathrm{~mL}, 3.47 \mathrm{mmol})$ was added dropwise. The reaction mixture was stirred at $-78{ }^{\circ} \mathrm{C}$ until completion (TLC monitoring). Then, it was quenched with a saturated aqueous solution of ammonium chloride and the crude product was extracted with $\mathrm{CH}_{2} \mathrm{Cl}_{2}(3 \mathrm{x}$ $10 \mathrm{~mL}$ ). The combined organic layers were dried with anhydrous $\mathrm{Na}_{2} \mathrm{SO}_{4}$, filtered, and evaporated. The crude product was purified by flash chromatography on silica gel (eluent: petroleum ether/ethyl acetate, 7/1).

(3R,4aS,8aS,1'S)-3-Methyl-4-(1-phenylethyl)-octahydro-benzo[1,4]oxazin-2-one (9). Crystalline solid $(80 \%$ yield $), \mathrm{mp} 172-173{ }^{\circ} \mathrm{C} ;[\alpha]_{\mathrm{D}}{ }^{28}=-21.5\left(\mathrm{c}=1.13, \mathrm{CHCl}_{3}\right) ;{ }^{1} \mathrm{H} \mathrm{NMR}: \delta 0.96$ $(\mathrm{d}, J=7.36 \mathrm{~Hz}, 3 \mathrm{H}), 1.33(\mathrm{~m}, 3 \mathrm{H}), 1.35(\mathrm{~d}, J=6.9 \mathrm{~Hz}, 3 \mathrm{H}), 1.50(\mathrm{~m}, 1 \mathrm{H}), 1.78(\mathrm{~m}, 1 \mathrm{H}), 1.87$ (m, $1 \mathrm{H}), 2.21-2.33(\mathrm{~m}, 2 \mathrm{H}), 2.66(\mathrm{~m}, 1 \mathrm{H}), 3.41(\mathrm{q}, J=7.36 \mathrm{~Hz}, 1 \mathrm{H}), 4.13(\mathrm{q}, J=6.9 \mathrm{~Hz}, 1 \mathrm{H}), 4.19$ $(\mathrm{m}, 1 \mathrm{H}), 7.22-7.45(\mathrm{~m}, 5 \mathrm{H}) ;{ }^{13} \mathrm{C} \mathrm{NMR}: \delta 10.1,23.1,24.1,24.1,31.5,32.1,52.3,56.1,59.7,78.1$, 127.1, 128.2, 128.3, 142.7, 174.3. Anal. Calcd for $\mathrm{C}_{17} \mathrm{H}_{23} \mathrm{NO}_{2}$ (273.17): C, 74.69; $\mathrm{H}, 8.48$. Found: C, 74.36; H, 8.57.

(3S,4aR,8aR,1'S)-3-Methyl-4-(1-phenylethyl)-octahydro-benzo[1,4]oxazin-2-one (9). Crystalline solid (60\% yield), mp 98-100 ${ }^{\circ} \mathrm{C} ;[\alpha]_{\mathrm{D}}{ }^{28}=+75.3\left(\mathrm{c}=1.8, \mathrm{CHCl}_{3}\right) ;{ }^{1} \mathrm{H} \mathrm{NMR}: \delta 0.9-1.42(\mathrm{~m}, 4 \mathrm{H}), 1.46$ $(\mathrm{d}, J=7.4 \mathrm{~Hz}, 3 \mathrm{H}), 1.47(\mathrm{~d}, J=6.8 \mathrm{~Hz}, 3 \mathrm{H}), 1.61(\mathrm{~m}, 1 \mathrm{H}), 1.74(\mathrm{~m}, 1 \mathrm{H}), 2.07(\mathrm{~m}, 2 \mathrm{H}), 2.53(\mathrm{~m}$, $1 \mathrm{H}), 3.85$ (q, $J=7.4 \mathrm{~Hz}, 1 \mathrm{H}), 4.04$ (q, $J=6.8 \mathrm{~Hz}, 1 \mathrm{H}), 4.13(\mathrm{~m}, 1 \mathrm{H}), 7.21-7.34(\mathrm{~m}, 5 \mathrm{H}) ;{ }^{13} \mathrm{C}$ NMR: $\delta 18.3,23.9,23.8,23.9,31.3,31.7,54.6,58.3,59.1,78.7,127.3,128.4,128.5,142.4$, 173.4. HRMS (FAB) $\mathrm{m} / \mathrm{z}$ found $274.1799[\mathrm{M}+\mathrm{H}]^{+}$, calcd for $\mathrm{C}_{17} \mathrm{H}_{24} \mathrm{NO}_{2} 274.1807$.

(3R,5S,1'S)-3-Methyl-5-phenyl-4-(1-phenylethyl)-1,4-oxazin-2-one (10). Crystalline solid (34\% yield), mp 129-130 ${ }^{\circ} \mathrm{C} ;[\alpha]_{\mathrm{D}}{ }^{28}=-40.5\left(\mathrm{c}=1.14, \mathrm{CHCl}_{3}\right) ;{ }^{1} \mathrm{H} \mathrm{NMR}: \delta 1.12(\mathrm{~d}, J=7.4 \mathrm{~Hz}$, $3 \mathrm{H}), 1.38$ (d, $J=6.8 \mathrm{~Hz}, 3 \mathrm{H}), 3.64$ (q, $J=7.4 \mathrm{~Hz}, 1 \mathrm{H}), 3.92$ (q, $J=6.8 \mathrm{~Hz}, 1 \mathrm{H}), 4.24(\mathrm{~m}, 3 \mathrm{H})$, 7.25-7.60 (m, 10H); ${ }^{13} \mathrm{C}$ NMR: $\delta$ 10.0, 23.3, 51.8, 56.9, 60.4, 70.3, 127.2, 128.0, 128.1, 128.1, 
128.5, 128.9, 138.8, 141.8, 173.5. HRMS $(\mathrm{FAB}) \mathrm{m} / \mathrm{z}$ found $318.1476[\mathrm{M}+\mathrm{Na}]^{+}$, calcd for $\mathrm{C}_{19} \mathrm{H}_{21} \mathrm{NO}_{2} \mathrm{Na} 318.1409$.

(3RS,6R,1'S)-3-Methyl-5-phenyl-4-(1-phenylethyl)-1,4-oxazin-2-one (11). Diastereomeric mixture in a 57:43 ratio (60 \% yield). ${ }^{1} \mathrm{H}$ NMR: $\delta 1.29(\mathrm{~d}, J=6.6,3 \mathrm{H}), 1.36(\mathrm{~d}, J=6.6 \mathrm{~Hz}, 3 \mathrm{H})$, $1.40(\mathrm{~d}, J=6.8 \mathrm{~Hz}, 3 \mathrm{H}), 1.62(\mathrm{~d}, J=6.8 \mathrm{~Hz}, 3 \mathrm{H}), 2.54$ (dd, $J=3.6 \mathrm{~Hz}, 1 \mathrm{H}), 2.83$ (dd, $J=3.0 \mathrm{~Hz}$, $1 \mathrm{H}), 2.92(\mathrm{~m}, 1 \mathrm{H}), 3.07(\mathrm{~m}, 1 \mathrm{H}), 3.7(\mathrm{~m}, 2 \mathrm{H}), 3.83(\mathrm{q}, J=6.6 \mathrm{~Hz}, 1 \mathrm{H}), 4.96(\mathrm{q}, J=6.8 \mathrm{~Hz}, 1 \mathrm{H})$, $5.33(\mathrm{dd}, J=3.0 \mathrm{~Hz}, 1 \mathrm{H}), 5.47(\mathrm{dd}, J=3.6 \mathrm{~Hz}, 1 \mathrm{H}), 7.20-7.45(\mathrm{~m}, 20 \mathrm{H}) ;{ }^{13} \mathrm{C}$ NMR: $\delta 11.0$, 13.5, 17.1, 21.6, 47.1, 49.0, 55.1, 56.0, 57.1, 58.4, 80.1, 80.5, 125.7, 125.9, 127.1, 127.2, 127.4, 127.6, 128.2, 128.3, 128.5, 128.7, 137.8, 138.0, 142.0, 142.1, 172.2, 173.1. HRMS (FAB) m/z found $296.1646[\mathrm{M}+\mathrm{H}]^{+}$, calcd for $\mathrm{C}_{19} \mathrm{H}_{22} \mathrm{NO}_{2} 296.1650$.

(3S,5S,6R,1'S)-3-Methyl-5,6-diphenyl-4-(1-phenylethyl)-1,4-oxazin-2-one (12). Crystalline solid (59\% yield), mp $138-140{ }^{\circ} \mathrm{C} ;[\alpha]_{\mathrm{D}}{ }^{28}=-50.0\left(\mathrm{c}=1.14, \mathrm{CHCl}_{3}\right) ;{ }^{1} \mathrm{H}$ NMR: $\delta 1.15(\mathrm{~d}, J=6.6$ $\mathrm{Hz}, 3 \mathrm{H}), 1.29(\mathrm{~d}, J=7.0 \mathrm{~Hz}, 3 \mathrm{H}), 3.9(\mathrm{q}, J=6.6 \mathrm{~Hz}, 1 \mathrm{H}), 4.13(\mathrm{q}, J=6.96 \mathrm{~Hz}, 1 \mathrm{H}), 4.34(\mathrm{~d}, J=$ $2.56 \mathrm{~Hz}, 1 \mathrm{H}), 5.89(\mathrm{~d}, J=2.96 \mathrm{~Hz}, 1 \mathrm{H}), 6.51-7.42(\mathrm{~m}, 15) ;{ }^{13} \mathrm{C}$ NMR: $\delta 18.9,22.8,56.9,59.7$, 63.6, 82.0, 126.4, 127.5, 127.7, 127.9, 128.1, 128.5, 129.1, 135.9, 136.1, 143.5, 165.9. HRMS (FAB) $\mathrm{m} / \mathrm{z}$ found $372.1974[\mathrm{M}+\mathrm{H}]^{+}$, calcd for $\mathrm{C}_{25} \mathrm{H}_{26} \mathrm{NO}_{2} 372.1964$.

\section{Acknowledgments}

We are grateful to SEP-PROMEP (Project No. 103.5/03/1156) and SEP-CONACyT for financial support (Projects No. 33023-E and 39500-2002), and to Professor Patrick Walsh (UPenn) for the high resolution mass spectra. We are also grateful to Roberto Melgar, Gabriela Ávila, Marcos Hernández, and Maribel Vázquez for their helpful comments.

\section{References and Notes}

1. (a ) Williams, R. M.; Sinclair, P. J.; Zhai, D.; Chen, D. J. Am. Chem. Soc. 1988, 110, 1547. (b) Dellaria, J. F.; Santarsiero, B. D. J. Org. Chem. 1989, 54, 3916. (c) Williams, R. M.; Fegrey, G. J. J. Am. Chem. Soc. 1991, 113, 8796. (d) Alami, A.; Calmes, M.; Daunis, J.; Escale, F.; Jaquier, R.; Roumestant, M.-L.; Viallefont, P. Tetrahedron: Asymmetry 1991, 2, 567. (e) Cativiela, C.; Diaz-de-Villegas, M. D.; Gálvez, J. A. Tetrahedron: Asymmetry 1992, 3, 567. (f) Chinchilla, R.; Falvello, L. R.; Galindo, N.; Nájera, C. Angew. Chem., Int. Ed. 1997, 36, 995.

2. See, for example: (a) Chinchilla, R.; Falvello, L. R.; Galindo, N.; Nájera, C. J. Org. Chem. 2000, 65, 3034. (b) Agami, C.; Couty, F.; Puchot-Kaudori, C. Synlett 1997, 449.

3. See, for example: (a) Jain P. R.; Williams, R. M. J. Org. Chem. 2002, 67, 6361. (b) Jain, P. R.; Albrencht, B. K.; De Mong, D. E.; Williams, R. M. Org. Lett. 2001, 26, 4287. (c) Agami, C.; Comesse, S.; Kadouri-Puchot, C. J. Org. Chem. 2000, 65, 4435. 
4. (a) Devine, P. N.; Foster, B. S.; Grabowski, E. J. J.; Reider, P. J. Heterocycles 2002, 58, 119. (b) Solladié-Cavallo, A.; Sedy, O.; Salisova, M.; Schmitt, M. Eur. J. Org. Chem. 2002, 3042 .

5. (a) Agami, C.; Comesse, S.; Kadouri-Puchot, C. J. Org. Chem. 2002, 67, 2424. (b) Santes, V.; Ortíz, A.; Santillán, R.; Gutiérrez, A.; Farfán, N. Synth. Commun. 1999, 29, 1277.

6. (a) Juaristi, E.; Escalante, J.; León-Romo, J. L.; Reyes, A. Tetrahedron: Asymmetry 1998, 9, 715. (b) Juaristi, E.; León-Romo, J. L.; Reyes, A. Escalante, J. Tetrahedron: Asymmetry 1999, 10, 2441.

7. (a) Overmann, L. E.; Flippin, L. A. Tetrahedron Lett. 1981, 22, 195. (b) Pracejus, H.; Pracejus, G.; Costisella, B. J. Prak. Chem. 1987, 329, 235.

8. Anaya de Parrodi, C.; Juaristi, E.; Quintero, L. An. Quím. Int. Ed. 1996, 92, 400.

9. Iuliano, A.; Pini, D.; Salvadori, P. Tetrahedron: Asymmetry 1995, 6, 739.

10. Alcalde, B.; De la Pradilla, R. F.; López-Mardomingo, C.; Pérez-Ossorio, R.; Plumet, J. J. Org. Chem. 1981, 46, 3234.

11. Atomic coordinates for all structures reported in this paper have been deposited with the Cambridge Crystallographic Data Centre. The coordinates can be obtained, on request from the Director, Cambridge Crystallographic Data Centre, 12 Union Road, Cambridge CB2 1EZ, UK. Deposition numbers: CCDC 217838, CCDC 217839, CCDC 217840, CCDC 217841.

12. Program Hyperchem for Windows and NT 5.0 (Hypercube, Inc).

13. Diastereoselectivity, ds, refers to the percentage of the most abundant diastereomer, see: Juaristi, E. Introduction to Stereochemistry and Conformational Analysis, Wiley: New York, 1991, pp 129-130.

14. While absolute values of AM1 calculated energies are not expected to be accurate, this semiempirical method has been to adequately reproduce relative energies of organolithium compounds. See: (a) Dewar, M. J. S.; Zoebisch, E. G.; Healy, E. F.; Stewart, J. J. P. J. Am. Chem. Soc. 1985, 107, 3902. (b) Schleyer, P. v. R. Encyclopedia of Computational Chemistry; John Wiley and Sons: New York, 1998; Vol.1, p 8. 